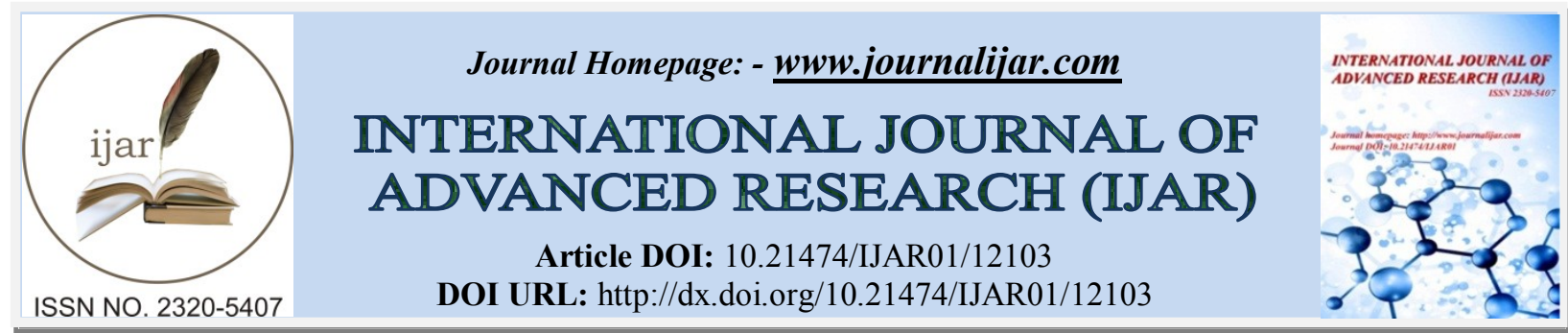

RESEARCH ARTICLE

\title{
THE SIGNIFICANCE OF CEA AND CA15-3 IN EARLY BREAST CANCER; ITS ASSOCIATION WITH CLINICO PATHOLOGICAL CHARACTERISTICS A SINGLE-CENTER STUDY IN MADINAH, SAUDI ARABIA
}

\author{
Nasser Mulla ${ }^{1}$, Yara Bin Saad ${ }^{2}$, Jamlaa Alharbi ${ }^{2}$ and Salwa Alalwi ${ }^{2}$ \\ 1. Medical Oncology Consultant, Assistant Professor, College Of Medicine, Taibah University. \\ 2. Internal Medicine Residents, King Fahad Hospital, Ministry of Health, Madinah, Saudi Arabia.
}

\section{Manuscript Info}

\section{Manuscript History}

Received: 25 September 2020

Final Accepted: 28 October 2020

Published: November 2020

Key words:-

Breast Cancer, Tumor Marker, CEA, CA15-3

\begin{abstract}
Background and Aims: The utility of measuring carcinoembryonic antigen (CEA) and cancer antigen 15-3 (CA15-3) levels in patients with breast cancer remains controversial. The present study aims to investigate the association between tumor markers [cancer antigen 15-3 (CA 15-3) and carcinoembryonic antigen (CEA)] and clinicopathological characteristics.

Methods: Serum preoperative CEA and CA 15-3 concentration levels were measured in a total of 135 breast cancer patients. The association of tumor markers levels with clinicopathological characteristics were analyzed.

Results: Elevated serum levels of CEA and CA15-3 were identified in $26(19.26 \%)$ and $20(14.8 \%)$ patients, respectively. Increased CEA levels were not associated with age, histological type, tumor size, tumor grade, breast cancer stages, or breast cancer types. CA15-3 levels were positively correlated with the size of the primary tumor $(\mathrm{P}<0.026)$ but had no significant correlation with the other factors.

Conclusions: The elevation of CA 15-3 was positively correlated with the size of the primary tumor.
\end{abstract}

Copy Right, IJAR, 2020,. All rights reserved.

\section{Introduction:-}

Globally, breast cancer is the most frequently diagnosed cancer among Women, and it is also considered the leading cause of cancer-related deaths between 40 and 49 females.(1)

Its early detection and diagnosis has an impact on the expected survival. The most commonly used prognostic indicators in breast cancer include patient characteristics (age and menstrual status), tumor characteristics (tumor size, node status, and TNM stage), tumor tissue markers (estrogen receptor, progesterone receptor, HER-2 and ki-67 status), and genetic markers (BRCA1/2). $(2,3)$

Tumor tissue markers play an important role in achieving this goal, the field of marker discovery is rapidly growing, leading to better understanding (1).

The prognostic importance of levels of preoperative CEA and CA15-3 in breast cancer has received significant interest in recent years. Studies have shown that preoperative levels of CEA combined with levels of CA15-3 can provide valuable knowledge for breast cancer diagnosis and treatment. Nonetheless, the prognostic importance of 
CEA and CA15-3 preoperative rates for breast cancer remains uncertain (4-6). The European Group on Tumor Markers has also proposed that the CEA and CA15-3 levels are used to determine prognosis, early detection of disease progression and monitoring of breast cancer treatment (7). Nevertheless, Maric et al. recently investigated the impacts of serum tumor markers in breast cancer and pointed to conflicting results of their prognostic value (8). As a result, the guidelines of the American Society of Clinical Oncology (ASCO) do not actually promote the usage of serum CA 15-3 and CEA for screening, diagnosis, staging or frequent evaluation of breast cancer patients during primary therapy $(9,10)$.

We therefore performed a retrospective review of the clinicopathological data of women with breast cancer to investigate the relationships between CEA and CA15-3 levels of preoperative plasma and the traditional clinicopathological prognostic factors.

\section{Materials and Methods:-}

Study Subjects and inclusion criteria:

We performed a retrospective analysis of data from breast cancer patients treated at King Fahad Hospital In Almadinah Almonawarah between January 2016 and December 2019.For research purposes, all participants involved in the study gave a written informed consent to the use of their medical record, and it is approved by ethical committee.

Criteria for inclusion were: female; unilateral or bilateral invasive breast cancer; mastectomy or breast-conserving surgery; levels of CEA and CA15-3 were determined prior to surgery; tumor completely removed by surgery without residual tumor as indicated by pathological evaluation, appropriate adjuvant chemotherapy, adjuvant radiotherapy and endocrine therapy administered based on international guidelines; complete results of estrogen receptor (ER), progesterone receptor (PR), HER2 and histologic grade; and no sign of metastasis or secondary cancer in other organs at diagnosis. A total 136 patients were eligible for this study.

\section{Measurement of CEA and CA15-3 levels and immunohistochemical evaluation:} Baseline CEA and CA15-3 data were obtained preoperatively for 136 patients.

The CEA and CA15-3 cut-off values were $3.0 \mathrm{ng} / \mathrm{mL}$ and $32 \mathrm{U} / \mathrm{mL}$ respectively, and if the value was above or below the cut-off value, the marker value was deemed either positive or negative.

ER and PRpositive have been defined as tumors with nuclear-stained cells of $>1 \%$. A $2+$ score from the immunohistochemical assessment indicated HER2-positivity and was confirmed using a fluorescence in situ hybridization (FISH) test for HER2.

The molecular subtypes were classified into three groups as follows: hormone receptor positive (ER+and/or PR+); HER2 positive (ER-and PR-, HER2+); and triple-negative (ER-and PR-,HER2-) according to the molecular subtype consensus of the St.

\section{Ethics Statement:}

Ethical approval will be taken from the ethical committee of king Fahad hospital.

Informed consent will be taken verbally from participant patients after explaining the aim of study and taking their agreement and they have full right to refuse the contribution.

The contribution in the study has no potential harms to the participants.

Privacy and confidentiality to the Participants in the study and their organizations will be assured as the data from each participant will be taken individually and will not be named in subsequent write ups and will not submitted for publication.

\section{Statistical analysis:}

Using the statistical analysis package SPSS v.26, all data is analyzed. Chi-Square tests was used to analyze the differences between the qualitative data. The stepwise Cox Regression Analysis included factors that were significant in the univariate analysis. P values inferior to 0.05 have been considered significant. 


\section{Results:-}

The patient characteristics:

There were 220 patients between 2016 and 2019 diagnosed with breast cancer, 136 met the criteria for inclusion in the study. Table(1) displays the clinicopathological features of the patients. The median age of onset of illness was 52 (range, 27-90 years). The 131 cases is pathologically listed as invasive ductal carcinoma. For all the cases, 90 were listed as positive hormone receptor, 34 as positive HER2 and 28 as triple-negative. 132 were classified as histologic grade II and above.

Relationship between clinic pathological characteristics and serum CEA and CA15-3 levels at baseline: Table 1 shows the relationships between the levels of these serum tumor markers (CEA and CA15-3) at baseline and the clinic pathological characteristics.

In the CEA-high subset, the higher frequencies were observed for age $>35$, Histological type IDC, tumor size $>2 \mathrm{~cm}$, Tumor grade II/III, Stage 2 and Hormone receptor-positive Breast cancer, but none of these frequencies was statistically significant. This indicates that there is no relationship between the levels of CEA and any of the aforementioned clinic pathological characteristics.

While in the CA15-3- high subset, significantly higher frequencies were observed only for larger tumor size $(\mathrm{P}=0.026)$, reflecting a relationship between elevation $\mathrm{CA} 15-3$ and tumor size. The other clinic pathological characteristics including; age > 35, Histological type IDC, Tumor grade II/III, Breast cancer in the second stage, and Hormone receptor-positive Breast cancer had higher frequencies in the CA15-3- high subset, but these frequencies were not statistically significant.

The analysis indicated that elevation of CA15-3 was positively correlated with the size of the primary tumor $(\mathrm{P}=0.026)$.

Table 1:- Clinicopathological characteristics of breast cancers according to CEA or CA15-3 levels.

\begin{tabular}{|c|c|c|c|c|c|c|}
\hline \multirow[t]{2}{*}{ Characteristics } & \multicolumn{2}{|l|}{ CEA } & \multirow[t]{2}{*}{ P-value } & \multicolumn{2}{|l|}{ CA15-3 } & \multirow[t]{2}{*}{ P-value } \\
\hline & $\begin{array}{l}\text { Normal } \\
(\mathrm{n}=109)\end{array}$ & $\begin{array}{l}\text { High } \\
(\mathrm{n}=26)\end{array}$ & & $\begin{array}{l}\text { Normal } \\
(\mathrm{n}=115)\end{array}$ & $\begin{array}{l}\text { High } \\
(\mathrm{n}=20)\end{array}$ & \\
\hline \multicolumn{7}{|l|}{ Age } \\
\hline$\leq 35$ & 11 & 1 & \multirow[t]{2}{*}{.315} & 10 & 2 & \multirow[t]{2}{*}{.850} \\
\hline$>35$ & 98 & 25 & & 105 & 18 & \\
\hline \multicolumn{7}{|l|}{ Histological type } \\
\hline IDC & 106 & 25 & \multirow[t]{3}{*}{.428} & 111 & 20 & \multirow[t]{3}{*}{699} \\
\hline ILC & 1 & 1 & & 2 & 0 & \\
\hline Others & 2 & 0 & & 2 & 0 & \\
\hline \multicolumn{7}{|l|}{ Tumor size } \\
\hline$<2 \mathrm{~cm}$ & 14 & 5 & \multirow[t]{2}{*}{.400} & 13 & 6 & \multirow{2}{*}{$.026^{*}$} \\
\hline$>2 \mathrm{~cm}$ & 95 & 21 & & 102 & 14 & \\
\hline \multicolumn{7}{|l|}{ Tumor grade } \\
\hline I & 3 & 0 & \multirow{2}{*}{.392} & 3 & 0 & \multirow[t]{2}{*}{465} \\
\hline II/III & 106 & 26 & & 112 & 20 & \\
\hline \multicolumn{7}{|l|}{ Lymph Node } \\
\hline Negative & 58 & 13 & \multirow{2}{*}{.768} & 61 & 10 & \multirow{2}{*}{.801} \\
\hline Positive & 51 & 13 & & 54 & 10 & \\
\hline \multicolumn{7}{|l|}{ Breast ca stages } \\
\hline I & 14 & 4 & \multirow[t]{3}{*}{.118} & 17 & 1 & \multirow{3}{*}{.493} \\
\hline II & 72 & 21 & & 78 & 15 & \\
\hline III & 23 & 2 & & 20 & 4 & \\
\hline \multicolumn{7}{|l|}{ Breast cancer types } \\
\hline receptor & 76 & 14 & \multirow[t]{2}{*}{.495} & 79 & 11 & \multirow[t]{2}{*}{.735} \\
\hline HER2 positive & 27 & 7 & & 28 & 6 & \\
\hline
\end{tabular}




\begin{tabular}{l|l|l|l|l|l|l|}
\hline Triple negative & 21 & 7 & 24 & 4 & \\
Hint: carcinoembryonic antigen (CEA) normal range in our lab is 0-3 ng/mlcancer antigen 15-3 (CA15-3) normal
\end{tabular}

15-3 (CA15-3) normal range in our lab is $0-32 \mathrm{IU} / \mathrm{ML}$

IDC invasive ductal carcinoma, ILC invasive lobular carcinoma.

Figure 1 showes the relationship between CA15-3 and Tumor size. It's clear that at the case of tumor size $>2 \mathrm{~cm}$, the frequencies of CA15-3 levels were higher than frequencies in the case of tumor size $<2 \mathrm{~cm}$. This indicates the direct relationship between tumor size and CA15-3 levels.

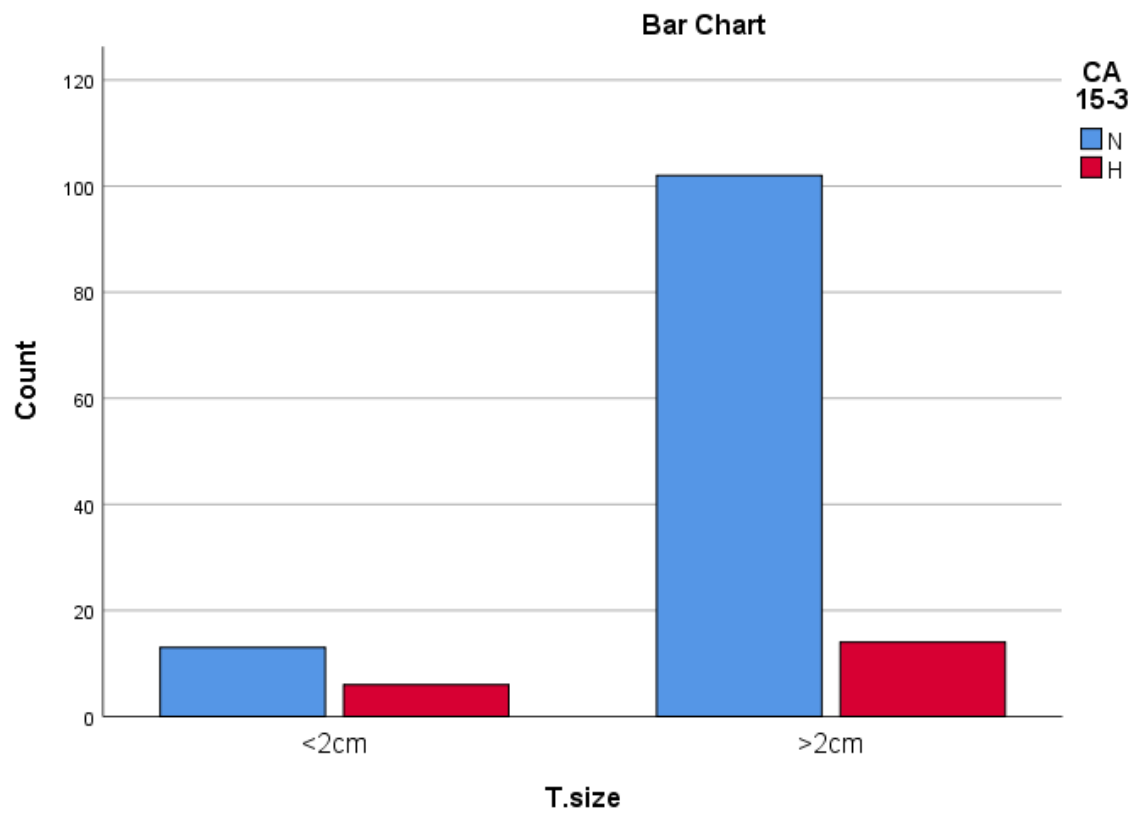

Fig1:- The relationship between CA15-3 and Tumor size.

\section{Discussion:-}

A major cause of death for women is breast cancer. Current oncology research focuses on identifying and validating novel biomarkers for early cancer diagnosis to improve care.

Traditional prognostic factors, such as axillary lymph node status, tumor size, histology grade, hormone receptor expression, and HER2 expression status, multigene assay and gene expression profiling have been spotlighted. All these factors require tissue samples. But at the other hand, serum is easily accessible and soluble circulating tumor markers will be ideal candidates for predicting outcome and following the course of treatment if found to be accurate prognostic factors. It is simple, objective, reproducible, and cost-effective to calculate markers. The most widely observed tumor markers for breast cancer have been serum CA 15-3 and CEA. Measuring markers is simple, objective, reproducible, and cost-effective.

CA 15-3 has been the most widely used marker in the last two decades in breast cancer patients for neoadjuvant chemotherapy response and prognosis along with other parameters because of its high sensitivity and specificity [11]. However, the significance of preoperative CA 15.3 levels is not well known [12]. A few studies have supported an association between high preoperative CA 15.3 and tumor burden and nodal status), and others have not $[13,14]$.

The results of this study including 135 women with breast cancer showed that preoperative CA15-3 levels were correlated with tumor size. Correlation of preoperative serum CA 15.3 levels with primary tumor size in patients with breast cancer has been demonstrated in Western data, however, the impact of preoperative CA 15.3 levels in Middle Eastern women with breast cancer has not been well studied $[17,18]$. The preoperative serum CEA and CA 15-3 levels are associated with the tumor size and lymph node metastasis which represents tumor burden [15,16] and 
significantly higher levels of CEA and CA 15-3 were seen in patients with advanced disease than in those with locoregional breast cancer [19]. The current study demonstrated the association of higher levels of CA 15-3 with tumor size.

Although the association of tumor markers and tumor biological factors is not well established, the serum CA 15-3 level was related to the tumor size in agreement with the study by Molina et al. [20,21]. A Meta-Analysis of 12,993 Patients demonstrated that higher rate of CA15-3 and CEA rise were correlated with tumor burden as larger tumor size, lymph node metastasis, and advanced TNM stages [22].

Previous studies have recorded variable findings on the prognostic value of CA 15-3 and CEA; the variability is due to limited sample size, short follow-up and variable cut-off values.; small sample size, short follow-up, and variable cut-off values contribute to the inconsistency. Duffy et al. [23] reported that a cut-off value of CA 15-3 might influence both the result and prognostic impact.

Our study is one of few studies that investigate the significance of CEA and CA15-3 and its association with clinicopathological characteristics of breast cancer patients in Saudi Arabia. It has some limitations. First, it is of small sample size. Second, it misses other prognostic clinicopathological characteristics such as Ki67.

In conclusion, elevated preoperative CA15-3 level is with tumor size. For other potential national surveys, this research will serve as a basis.. However, analysis of larger cohort of patients is needed to validate new prognostic markers in breast cancer.

\section{References:-}

1. Kesson EM, Allardice GM, George WD, et al. Effects of multidisciplinary team working on breast cancer survival: retrospective, comparative, interventional cohort study of 13722 women. BMJ 2012; 344:e2718.

2. Goldhirsch, W. C. Wood, R. D. Gelber et al., "Progress and promise: highlights of the international expert consensus on the primary therapy of early breast cancer 2007," Annals of Oncology, vol. 18, no. 7, pp. 11331144, 2007.

3. J. S. Ross, J. A. Fletcher, G. P. Linette et al., "The HER-2/neu gene and protein in breast cancer 2003: biomarker and tar- get of therapy," The Oncologist, vol. 8, no. 4, pp. 307-325, 2003

4. LeeJS, ParkS, ParkJM,ChoJH,KimSI,ParkBW.ElevatedlevelsofpreoperativeCA15-3andCEA serumlevels have independently poor prognostic significance in breast cancer. Ann Oncol 2013; 24:1225-1231. doi: 10.1093/annonc/mds604 PMID: 23230137

5. WuSG,HeZY,ZhouJ,SunJY,LiFY,LinQetal.SerumlevelsofCEAandCA15-3 in differentmolecular subtypes and prognostic value in Chinese breast cancer. Breast 2014; 23:88-93. doi: 10.1016/j. breast.2013.11.003 PMID: 24291374

6. PedersenAC,SorensenPD,JacobsenEH,MadsenJS,BrandslundI.Sensitivity of CA15-3,CEA and serumHER2intheearlydetectionof recurrenceof breast cancer. Clin Chem Lab Med2013; 51:15111519. doi: 10.1515/cclm-2012-0488 PMID: 23403727

7. MolinaR,BarakV,vanDalenA,DuffyMJ,EinarssonR,GionMetal.TumormarkersinbreastcancerEuropeanGroupon TumorMarkersrecommendations.Tumour Biol2005; 26:281-293.PMID: 16254457

8. MaricP,OzreticP,LevanatS,OreskovicS, AntunacK,Beketic-OreskovicL. Tumor markers in breast cancerevaluation of their clinical usefulness. Coll Antropol 2011; 35:241-247.

9. CardosoF,SaghatchianM,ThompsonA,RutgersE.Inconsistentcriteria used in AmericanSociety of Clinical Oncology 2007 update of recommendationsfor the use of tumor markers in breast cancer. J Clin Oncol 2008; 26:2058-2059; author reply 2060-2051. doi: 10.1200/JCO.2007.15.6638 PMID: 1842106314.

10. HarrisL,FritscheH, MennelR,NortonL, RavdinP, TaubeSetal.AmericanSocietyof Clinical Oncology 2007updateofrecommendationsfor theuse of tumormarkersinbreast cancer. J Clin Oncol 2007; 25:5287-5312. PMID: 17954709

11. Miya T, Watanabe T, Adachi I, et al. Analysis of cytosol CA15-13, carcinoembryonic antigen, and estrogen and progesterone receptors in breast cancer tissues. Jpn J Cancer Res. 1992;83:171-177.

12. Duffy MJ, Catherine D, Rachel K, et al. High preoperative CA 15.3 concentrations predict adverse outcome in node-negative and node-positive breast cancer: study of 600 patients with histologically confirmed breast cancer. Clin Chem. 2004;50:559-563.

13. Sandri MT, Salvatici M, Botteri E, et al. Prognostic role of CA15.3 in 7942 patients with operable breast cancer. Breast Cancer Res Treat. 2012;132:317-326. 
14. Lumachi F, Brandes AA, Ermani M, Bruno G, Boccagni P. Sensitivity of serum tumor markers CEA and CA 15-13 in breast cancer recurrences and correlation with different prognostic factors. Anticancer Res. 2000;20:4751-4755.

15. Park BW, Oh JW, Kim JH, et al. Preoperative CA 15-3 and CEA serum levels as predictor for breast cancer outcomes. Ann Oncol. 2008;19(4):675-681. doi:10.1093/annonc/mdm538

16. Tampellini M, Berruti A, Gerbino A, et al. Relationship between CA 15-3 serum levels and disease extent in predicting overall survival of breast cancer patients with newly diagnosed metastatic disease. Br $\mathrm{J}$ Cancer. 1997;75(5):698-702. doi:10.1038/bjc. 1997.124

17. Alsaeed, Eyad Fawzi et al. "Elevated preoperative serum cancer antigen 15.3 levels are associated with reduced disease-free survival: a single-institution experience.” Breast cancer (Dove Medical Press) vol. 5 53-9. 26 Jul. 2013, doi:10.2147/BCTT.S48568

18. Elfagieh M, Abdalla F, Gliwan A, Boder J, Nichols W, Buhmeida A. Serum tumour markers as a diagnostic and prognostic tool in Libyan breast cancer. Tumour Biol. 2012;33(6):2371-2377. doi:10.1007/s13277-012-0500-6

19. Duffy MJ. Serum tumor markers in breast cancer: are they of clinical value?. Clin Chem. 2006;52(3):345-351. doi:10.1373/clinchem.2005.059832

20. Molina R, Auge JM, Farrus B, et al. Prospective evaluation of carcinoembryonic antigen (CEA) and carbohydrate antigen 15.3 (CA 15.3) in patients with primary locoregional breast cancer. Clin Chem. 2010;56(7):1148-1157. doi:10.1373/clinchem.2009.135566

21. Molina R, Augé JM, Escudero JM, et al. Evaluation of tumor markers (HER-2/neu oncoprotein, CEA, and CA 15.3) in patients with locoregional breast cancer: prognostic value. Tumour Biol. 2010;31(3):171-180. doi:10.1007/s13277-010-0025-9

22. Li, X., Dai, D., Chen, B., Tang, H., Xie, X., \& Wei, W. (2018). Clinicopathological and Prognostic Significance of Cancer Antigen 15-3 and Carcinoembryonic Antigen in Breast Cancer: A Meta-Analysis including 12,993 Patients. Disease markers, 2018, 9863092. https://doi.org/10.1155/2018/9863092

23. Duffy MJ, Duggan C, Keane R, et al. High preoperative CA 15-3 concentrations predict adverse outcome in node-negative and node-positive breast cancer: study of 600 patients with histologically confirmed breast cancer [published correction appears in Clin Chem. 2004 Jun;50(6):1111]. Clin Chem. 2004;50(3):559-563. doi:10.1373/clinchem.2003.025288. 